\title{
Coulisses
}

Revue de théâtre

40 | Hiver 2010

Jeux de rappels chez Marivaux

\section{La mauvaise foi des sincères et la censure de la vanité}

Beya Dhraïeff

\section{(2) OpenEdition}

1 Journals

Édition électronique

URL : https://journals.openedition.org/coulisses/661

DOI : 10.4000/coulisses. 661

ISSN : 2546-9460

Éditeur

Presses universitaires de Franche-Comté

\section{Édition imprimée}

Date de publication : 1 janvier 2010

Pagination : 68-82

ISBN : 978-2-84867-283-0

ISSN : $1150-594 X$

\section{Référence électronique}

Beya Dhraïeff, «La mauvaise foi des sincères et la censure de la vanité », Coulisses [En ligne], 40 | Hiver 2010, mis en ligne le 30 novembre 2016, consulté le 29 décembre 2022. URL : http://

journals.openedition.org/coulisses/661 ; DOI : https://doi.org/10.4000/coulisses.661

Ce document a été généré automatiquement le 29 décembre 2022.

Tous droits réservés 


\title{
La mauvaise foi des sincères et la censure de la vanité
}

\author{
Beya Dhraïeff
}

Pas assez de cynisme et pas assez de vertu. Nous n'avons ni l'énergie du mal, ni celle du bien.

Camus

1 Plusieurs critiques ont questionné les subterfuges développés par les personnages marivaudiens pour s'éluder. Dans une étude qui reste essentielle, Sanaker ${ }^{1}$ associe ainsi leurs perturbations langagières à leur confusion intime : en perdant le contrôle de leurs discours, ils dévoileraient les disjonctions intérieures qu'opère leur refus d'accepter leur être ou son évolution. Recourant aussi à la linguistique de l'énonciation, AnneMarie Paillet-Guth ${ }^{2}$ y joint l'éclairage de la philosophie existentialiste pour expliquer « la mauvaise foi interprétative » des amoureux de Marivaux et de Crébillon tandis que Michel Gilot ${ }^{3}$ souligne ingénieusement l'alliance insolite de la bonne et de la mauvaise foi dans la psyché de la Marquise de L'Heureux Stratagème. Plus récemment, John O'Neal ${ }^{4}$ invoque enfin l'épistémologie lockienne pour décrypter l'état de confusion d'Arlequin et de Silvia dans La Double Inconstance : le flou de leurs sentiments déteignant sur leurs idées, leur formulation s'en trouverait contrariée.

2 Ce travail s'inscrit dans une perspective sensiblement différente: la mauvaise foi ne perturbe pas uniquement le discours amoureux, elle me semble avant tout expliquer le fonctionnement de la conscience morale. L'analyse des Sincères le révèle et présente l'intérêt supplémentaire de mettre en abyme l'ensemble du théâtre marivaudien, en problématisant la possibilité et l'opportunité de la sincérité. La rigueur de construction de la pièce sert sa visée démonstrative et suggère, en filigrane, une stratégie opportune pour l'aborder. Trois moments dramatiques clés retiennent l'attention : l'exposition des personnages ; l'Epreuve du Miroir et la suspicion de la mauvaise foi ; la restauration du narcissisme par la fausse sincérité. 


\section{Exposition(s)}

3 Au lever du rideau, Frontin, valet d'Ergaste, et Lisette, suivante de la Marquise, rompent avec la tradition dramatique en se confiant leur mutuelle indifférence: Frontin serait amoureux de Marton, suivante d'Araminte, tandis que Lisette aurait donné son cœur à Dubois, valet de Dorante. Mus par la communauté de leur intérêt, les deux suivants décident alors de comploter pour rompre l'union d'Ergaste et de la Marquise, en reconstituant leurs couples primitifs : Ergaste / Araminte ; Dorante / La Marquise.

4 Forte de sa finesse d'esprit, Lisette commence par souligner que l'union provisoire de La Marquise et d'Ergaste se justifie par la communauté de leur aveuglement sur euxmêmes ${ }^{5}$ :

5 «Ils s'imaginent sympathiser ensemble à cause de leur prétendu caractère de sincérité. » L'usage du verbe "s'imaginer » et de l'épithète « prétendu » matérialise la distance critique à partir de laquelle Lisette évalue les convictions des Sincères. Sa lucidité omnisciente révèle l'écart existant entre la manière dont Ergaste et La Marquise se conçoivent et la vérité de leur être intime. Insinuant leur mauvaise foi, elle la présente comme l'origine de leurs rapports mystifiés. Leur illusion de sincérité conditionnerait leur sympathie fallacieuse; leur mensonge sur eux-mêmes fausserait leurs relations, en les entretenant dans le rêve d'une identité chimérique. Le terme "sympathie " me semble à comprendre ici dans son sens étymologique de «souffrir avec l'autre »; La Marquise et Ergaste souffriraient ensemble de leur différence avec les autres; ils se délecteraient de la souffrance agréable que leur procure leur sincérité élective. Lisette et Frontin décident de profiter de leur «marotte " pour les brouiller. Afin d'y arriver, ils se dévoilent et révèlent par ricochet aux spectateurs les arcanes des caractères de leurs maîtres.

6 Le dévoilement de La Marquise par Lisette s'effectue graduellement. Dans sa première tirade, la suivante expose la nature de sa maîtresse avant de se focaliser, dans la seconde, sur le comportement à adopter pour lui complaire. Lisette commence par dégager trois attributs définitoires du caractère de la Marquise - «vaine, envieuse et caustique » - avant de les illustrer par l'évocation de situations concrètes qui les révèlent. Sa « vanité » déterminerait son « envie » et sa causticité : la suivante dépeint sa maîtresse comme un être dont la propension à la médisance procède du narcissisme. Le rabaissement de l'autre conditionnerait la divinisation de soi. Un ample champ lexical parcourt la tirade de Lisette pour figurer la tyrannie de la Marquise : « ceux qui tremblent sous elle »; « les entretenir dans la crainte »; « elle prime»; «sa sujette»; « avec respect de ses bonnes grâces »; «c'est elle qui vous le permet »; «à elle »...

7 Au-delà de ses désirs d'omnipotence, la Marquise apparaîtrait profondément manipulable. Elle est agie par son « esprit qui la rend méchante » et la pousse à trahir son « cœur ». Sa malignité qui la défigure à son insu révèle sa spécificité par rapport à son modèle littéraire le plus immédiat, la Célimène moliéresque. Si l'esprit de l'héroïne du Misanthrope est certainement méchant, son cœur reste insondable : Alceste répète à maintes reprises qu' " on ne voit pas les cœurs ${ }^{6}$, Eliante signale sa méconnaissance d'elle-même ${ }^{7}$ et Célimène confirme indirectement ces propos en postulant le "grand aveuglement où chacun est pour soi $»^{8}$. Le dénouement même de la pièce ne dissipe pas le mystère qui plane sur une intériorité qu'aucun monologue n'expose dans sa pleine nudité. Dans sa conception de Célimène, Molière semble vouloir maintenir le 
questionnement sur sa nature profonde alors que, par la bouche de sa suivante, Marivaux postule une disjonction entre l'esprit et le cœur de la Marquise, même si la suite de la pièce ne confirme guère sa prétendue bonté ontologique.

Selon Lisette, la Marquise se servirait du langage pour se dissimuler son caractère. Elle s'aveugle sur elle-même en travestissant sa véritable nature par sa qualification : « elle appelle austérité de raison » " son caractère sec et formidable ». La Marquise se ment à elle-même sur elle-même mais elle ment aussi aux autres en leur demandant de comprendre son être sans qu'elle l'exprime. Les propos qu'on lui tient doivent émaner d'une science infuse de sa personnalité et non répondre à ses mots. La stratégie implicite utilisée par Lisette pour démasquer l'intériorité de la Marquise est celle que préconisait «le Voyageur dans le monde vrai » pour distinguer l'être du paraître : ne pas s'attacher à la teneur mais à « la tournure de (ses) discours ", à « l'air qu'(elle a) en parlant", à son "ton", à ses "gestes", "même (à) ses regards ". C'est en s'interrogeant sur l'incongruité de son «aigreur » et de sa «brusquerie » lorsqu'à sa demande, elle l'a expédiée un peu aux dépens des grâces, que la suivante aurait démêlé le premier principe de fonctionnement de sa maîtresse : sa duplicité langagière.

Le discours de la Marquise se caractérise par sa perversion, car il ne dit jamais ce qu'il a l'air de vouloir dire: il se veut inconsciemment performatif d'actes contraires à sa signification littérale. La Marquise pratique en effet la dérivation allusive : son langage ne formule pas directement ses désirs mais les insinue, par la multiplication de signes connexes, essentiellement non verbaux, qui permettent à son interlocutrice de deviner sa demande réelle. On en arrive ainsi à un singulier paradoxe : l'exigence de sincérité qu'elle clame s'avère un besoin de raffinement dans le mensonge. La conscience critique de Lisette nous révèle que sa maîtresse ne rechercherait pas la sincérité qu'elle croit souhaiter mais savourerait l'hypocrisie en franchise qui la conforte dans sa mauvaise foi.

Sommé de brosser complémentairement le portrait d'Ergaste, Frontin commence par le distinguer de la Marquise, en mettant en relief l'authenticité de sa sincérité avec autrui. Alors que la prétendue franchise de celle-ci camouflerait sa médisance, Ergaste «n'en veut à personne ", «il dit ce qu'il pense de tout le monde ": sa parole se conforme à sa pensée ; elle la traduit dans son intégralité. Il serait vraiment sincère dans ses discours. On serait tenté de le rapprocher de l'Alceste moliéresque. Même si c'est avec déformation, un épisode, relaté par Frontin, émanerait notamment tout droit de la biographie du misanthrope : celui du procès perdu. Mais il me semble significatif que le nom d'Ergaste ne soit que l'anagramme imparfaite d'Alceste. Le personnage marivaudien se différencie de son modèle présumé à deux niveaux complémentaires : sa conception de la sincérité et sa posture par rapport à la société.

11 La sincérité d'Alceste est à elle-même sa propre finalité. Il la prône au nom d'un idéal d'intégrité, d'une morale absolue ${ }^{10}$. Même s'il cherche à motiver la reconnaissance de sa différence ${ }^{11}$, être distingué lui apparaît comme un hommage dû, non tant à sa personne qu'à l'exigence de purisme qu'il incarne et qui le meut. L'applaudir, c'est admettre le bien fondé de sa quête existentielle et éthique. A l'inverse, Ergaste ne recherche pas la sincérité pour elle-même mais parce qu'elle constitue un moyen commode d'atteindre l'objectif de sa singularisation. Dans la tirade de Frontin, de multiples exemples illustrent cette manie qu'un champ lexical étendu dévoile : "se distinguer », " étonné », « autrement », « ne ressemble qu'à lui », « rare », « original », « extraordinaire »... 
Contrairement à celle d'Alceste, la sincérité d'Ergaste n'est nullement dictée par le respect d'un impératif moral. Frontin le souligne : son maître n'a cure d'être estimable. L'idéal qui régit ses actes n'est pas éthique : il est aristocratique, comme le prouve son rejet de ce qui serait «trop peuple». Sa quête de la singularité est une affirmation de son élection. Son choix de la sincérité avec les autres se double d'ailleurs d'une prédilection pour la médisance sur soi. Alors qu'Alceste est convaincu de ses qualités personnelles, Ergaste s'acharne à se discréditer, non par réelle conscience de son indignité comme il l'allègue, mais par excès de narcissisme comme le suggère Frontin. Il se conforme de la sorte au portait de l'eirôn que brosse Aristote dans son Ethique à Nicomaque $^{12}$ :

Les dissimulés (eirôn), qui ne parlent que pour minimiser leurs mérites, paraissent à première vue gens plus distingués (car ce n'est pas, pense-t-on, l'amour du gain qui inspire leurs paroles, mais la peur de l'exagération). Leur domaine à eux aussi, comme aux vantards (alazôn), ce sont les qualités dont on peut se faire gloire, mais eux, ils nient les posséder ; ainsi faisait Socrate. Mais aller jusqu’à nier posséder que des mérites qui ne sont que peu de chose et qu'on possède évidemment, c'est ce qu'on appelle un raffinement de fourberie, et cela prête plus facilement au mépris (c'est même quelquefois vantardise manifeste, comme l'est par exemple de s'habiller à la spartiate; car aussi bien que l'excès de richesse, la négligence exagérée du costume relève de la vantardise).

13 L'eirôn rejoint l'alazôn par un détour. Comme l'écrira l'Indigent philosophe ${ }^{13}$, «la modestie réelle et vraie n'est peut-être qu'un masque parmi les hommes ». Tandis qu'Alceste s'érige volontiers en modèle, Ergaste l'outrepasse en se voulant unique. Matérialisée par son abus de l'hyperbole et des superlatifs, sa fascination pour toutes les formes d'excès conditionne sa prétention à être inimitable jusque par les défauts réels ou chimériques dont il se targue. Pour reprendre un terme cher à Bakhtine, Ergaste serait un caractère carnavalesque par son obsession caricaturale de s'ériger à l'encontre de toutes normes pour prouver son exceptionnalité.

Le l'indifférenciation qui le tenaille émane de sa hantise de l'indifférence. Ergaste s'affirme comme un anti Alceste par son rapport à la société. A la misanthropie bien connue de ce dernier correspond et répond l'aliénation du personnage marivaudien par le regard social. Aliénation : le mot n'est pas trop fort. Ergaste se meut dans la société comme un acteur qui ne vit que des applaudissements de son public. Frontin signale que faire réagir les autres lui procure un sentiment de "triomphe"; "gagner (une) réputation» l'« enivre». Ergaste est un exhibitionniste doublé d'un voyeur : il ne se montre que pour jouir de l'effet du spectacle qu'il procure. Sa sincérité est une pose : sa sincérité n'est que le rôle de ses plus grands succès.

Ergaste accepte toutefois de jouer le rôle d'un public bienveillant pour un autre personnage : la Marquise. Mais il ne le fait que tant que celle-ci le lui rend bien. A la scène IV des Sincères, les protagonistes principaux s'exposent et exposent le rapport originel qui les unit. Ergaste stimule les débordements médisants de la Marquise. Il multiplie les questions, tantôt pour enclencher, tantôt pour relancer ses peintures satiriques. Il approuve ses critiques et cautionne ses portraits de son rire. Constante tout au long de la scène, l'hilarité du personnage motive le flux fielleux des propos de la Marquise. Il semble ainsi justifier ces mêmes reproches qu'Alceste adressa à la cohorte des soupirants de Célimène : " c'est (lui); et (ses) ris complaisants / (Qui) tirent de son esprit tous ces traits médisants $»^{14}$. Même si Ergaste ne médit jamais effectivement, il ne serait donc pas moins médisant, en esprit et en intention, que son interlocutrice. Il 
l'encourage dans son être pour se justifier son narcissisme : il l'applaudit pour se louer de sa singularité par rapport à ses objets de dérision.

Réciproquement, la Marquise recherche la connivence d'Ergaste pour se dissimuler sa malignité. "L'encens flatteur $»^{15}$ de son destinataire prétendument exemplaire lui permet de se croire juste et véridique, et non, caustique et méchante. Cependant ses médisances sur ses visiteurs lui constituent une toute autre image que celle qu'elle entend renvoyer. À la différence de Célimène dont les "portraits sont des miniatures » et les «tableaux des esquisses ${ }^{16}$, la Marquise des Sincères charge ainsi ses modèles au point d'effleurer la caricature, même si elle souligne paradoxalement qu'elle $n$ ' « exagère pas d'un mot ${ }^{17}$. Elle résume chacune de ses cibles dans un défaut principal : la fatuité du jeune homme à la mode, la niaiserie de la jeune mariée, la vénalité du cadet, l'embonpoint de la veuve et le verbiage du petit homme. Automatisant les vivants qu'elle observe, elle les rend d'autant plus ridicules qu'elle détaille complaisamment les manifestations de leur déficience majeure.

Dans la galerie des portraits qu'elle brosse, «le fat qui biaise» lui inspire incontestablement le plus de propos fielleux. Lui consacrant ses trois premières tirades alors qu'elle expédie notamment le babillard et le solennel porteur d'une "grande perruque » en une seule et même réplique, elle ne se contente pas de détailler ses ridicules; elle les mime $^{18}$ :

LA MARQUISE : Imaginez-vous qu'il n'a précisément qu'un objet dans la pensée, c'est de se montrer ; quand il rit, quand il s'étonne, quand il vous approuve, c'est qu'il se montre. Se tait-il, change-t-il de contenance, se tient-il sérieux, ce n'est rien de tout cela qu'il veut faire, c'est qu'il se montre; c'est qu'il vous dit: «Remarquez mes gestes et mes attitudes; voyez mes grâces dans tout ce que je fais, dans tout ce que je dis; voyez mon air fin, mon air leste, mon air cavalier, mon air dissipé ; en voulez-vous du vif, du fripon, de l'agréablement étourdi ? en voilà.» Il dirait volontiers à tous les amants : "N'est-il pas vrai que ma figure vous chicane? ", à leurs maîtresses : "Où en serait votre fidélité, si je voulais?", à l'indifférente : «Vous n'y tenez point; je vous réveille, n'est-ce pas?», à la prude: «Vous me lorgnez en dessous?", à la vertueuse: «Vous résistez à la tentation de me regarder? », à la jeune fille : «Avouez que votre cœur est ému ! » Il n'y a pas jusqu'à la personne âgée, qui, à ce qu'il croit, dit en elle-même en le voyant: "Quelle dommage que je ne sois plus jeune!»

18 Par sa figuration du vaniteux, la Marquise conjugue les talents d'acteur et de metteur en scène. Forte d'une lucidité comparable à celle qu'acquiert «le Voyageur dans le monde vrai", elle ne reproduit pas réellement ses actes et propos effectifs, elle déchiffre ses mines pour révéler ses pensées intimes. Ce faisant, elle dévoile l'intériorité que farde habituellement le jeu des simulacres sociaux.

La dextérité manifestée par le personnage marivaudien dans la représentation de la vanité doit cependant nous interpeller. La Marquise sait de quoi elle parle; sa complaisance dans la malveillance est proportionnelle à son implication par sa satire. Alors que les autres "originaux » lui restent en quelque sorte extérieurs par leurs défauts qu'elle se borne à accumuler, elle réussit à incarner le fat avec vraisemblance parce qu'elle "fait semblant de faire semblant $»^{19}$, comme l'écrira Marivaux après Dufresny $^{20}$. La Marquise pressent confusément son identité avec le personnage qu'elle critique. Son portrait féroce lui permet de se purger d'elle-même en se transformant en objet ridicule. Des termes non équivoques formulent son soupçon de sa réalité intérieure $^{21}$ : 
Un fat se doute toujours un peu qu'il l'est; et comme il a peur qu'on ne s'en doute aussi, il biaise, il est fat le plus modestement qu'il lui est possible, et c'est justement cette modestie-là qui rend sa fatuité sensible. clairvoyance ne lui apparaît toutefois pas absolue et constante, mais relative et intermittente. Un fat ne se bornerait pas à biaiser avec autrui, il louvoierait avec luimême pour se fuir. Comme l'écrivait Nicole ${ }^{22}$, «l'on [s]'évite en quelque sorte, parce qu'on ne [s']évite pas tout à fait. La vérité se fait toujours un peu de jour au travers de tous ces nuages dont on s'efforce de l'obscurcir. Il en passe toujours quelques rayons qui incommodent l'orgueil, et qui troublent ce faux repos qu'il s'efforce de se procurer.»

21 La Marquise est de mauvaise foi, c'est-à-dire qu'elle sait « en tant que trompeu(se) la vérité qui lui est masquée en tant qu'[elle] est trompée. Mieux encore, [elle ne sait] très précisément cette vérité [que] pour se la cacher plus soigneusement. ${ }^{23}$ Alors que le mensonge présuppose la dualité du trompeur et du trompé ; la mauvaise foi repose sur leur confusion au sein d'une même conscience. Comme le souligne Sartre ${ }^{24}$, « l'essence même de l'idée réflexive de 'se dissimuler' quelque chose implique l'unité d'un même psychisme et par conséquent une double activité au sein de l'unité, tendant d'une part à maintenir et à repérer la chose à cacher et d'autre part à la repousser et à la voiler ; chacun des deux aspects de cette activité est complémentaire de l'autre, c'est-à-dire qu'il l'implique dans son être. »

Cependant, dans le cas précis de la Marquise des Sincères, la tension entre révélation et occultation de soi ne s'incarne pas dans le mécanisme de la censure, comme le stipule le philosophe existentialiste ${ }^{25}$. Elle provient de la jonction de la mauvaise foi du personnage avec sa vanité. Pour Marivaux, l'amour-propre jouerait précisément le rôle que Sartre attribuera à la censure. Héritier des moralistes classiques, notre dramaturge conçoit le fonctionnement psychique de son héroïne à la lumière de leur représentation de l'intériorité humaine. On a déjà eu l'occasion de le suggérer : il s'inscrirait plus spécifiquement dans la continuité de Nicole. Dans un texte fondamental, De la connaissance de soi-même, ce dernier souligne en effet que la vanité de l'homme conditionne son ambivalence ${ }^{26}$ :

L'homme veut se voir, parce qu'il est vain. Il évite de se voir, parce que étant vain il ne peut souffrir la vue de ses défauts et de ses misères. Pour accorder donc ces désirs contraires, il a recours à un artifice digne de sa vanité, par lequel il trouve moyen de les contenter tous deux en même temps. C'est de couvrir d'un voile tous ses défauts, de les effacer en quelque sorte de l'image qu'il se forme de lui-même, et de n'y laisser que les qualités qui le peuvent relever à ses propres yeux. S'il ne les a pas effectivement, il se les donne par son imagination; et s'il ne les trouve pas dans son propre être, il les va chercher dans les opinions des hommes, ou dans les choses extérieures qu'il attache à son idée, comme si elles en faisaient partie; et par le moyen de cette illusion, il est toujours absent de lui-même et présent à lui-même : il se regarde continuellement, et il ne se voit jamais véritablement, parce qu'il ne voit au lieu de lui-même que le fantôme qu'il s'en est formé.

23

Le comportement paradoxal de la Marquise se conforme aux règles de fonctionnement de la psyché humaine qu'énonce Nicole. Déterminant son désir de se connaître, son amour-propre la pousse toutefois à ne se reconnaître que dans un portrait flatteur d'elle-même. Il fonde son besoin de se mystifier sur la réalité de son être intime. La Marquise recourt à autrui pour renforcer sa foi en la vérité de son image fantasmatique. Elle choisit plus spécifiquement Ergaste comme miroir car sa prétendue 
sincérité lui permet de croire à l'intégrité de sa quête de soi : le caractère dont il s'enorgueillit la confirme tant dans son illusion de clairvoyance que d'objectivité sur elle-même. Cependant, au moment même où la Marquise sollicite le franc témoignage d'Ergaste pour se juger, elle manœuvre, plus ou moins consciemment, pour ne s'entendre dire que ce qui peut lui agréer. Au début de la scène IV, elle loue son interlocuteur pour motiver ses louanges: «je n'ai encore vu qu'un homme vrai ; et en fait de femmes, je n'en connais pas. ${ }^{27}$ Louvoyant tant avec Ergaste qu'avec elle-même, la Marquise cède aux postulations antagonistes de la connaissance et de l'évitement de soi. La modestie factice qu'elle affiche est un raffinement de son amour-propre pour motiver l'éloge réciproque de sa sincérité. Afin d'y déterminer Ergaste sans qu'il s'en aperçoive et sans qu'elle-même s'en rende compte, elle le complimente mais drape sa flatterie d'impersonnalité pour qu'elle n'ait précisément pas l'air flatteur. Toutefois, en usant de la tournure restrictive, elle ne souligne que mieux l'exceptionnalité de son allocutaire par rapport au genre humain. La louange obviée de la sincérité d'Ergaste fonctionne ainsi comme le relais de sa confirmation implicite dans son idéal de singularisation. En justifiant son interlocuteur dans son fantasme sur lui-même, la Marquise motive avec succès la reconnaissance de sa vérité chimérique. Elle enclenche une émulation dans la complaisance que chacun d'eux manifeste à l'image mentale que l'autre se fait de lui-même.

Les deux personnages s'extasient d'autant plus volontiers sur leurs mérites mutuels que leur illusion d'identité leur procure le plaisir indirect de faire leur propre panégyrique. Pour chacun d'eux, louer son reflet dans l'autre, c'est se louer pour son reflet et offrir à son amour-propre l'opportunité savoureuse d'une expression biaisée. À la scène IV, la collusion inconsciente de la Marquise et d'Ergaste dans la fausse sincérité assure ainsi le triomphe de leur mauvaise foi qu'elle farde : premières dupes des rôles qu'ils se jouent, ils se confortent dans la confusion de leur apparence rêvée avec leur identité effective. Ils se retirent alors «bien satisfaits » l'un de l'autre mais, comme le suggère Frontin, « cela ne saurait durer $»^{28}$.

\section{L'Épreuve du Miroir et la suspicion de la mauvaise foi}

Leur retour, à la scène XII, enclenche la dégradation accélérée de leurs rapports. Sous couvert de sonder la sincérité de son amour, la Marquise interroge implicitement Ergaste sur la manière dont il se la représente : une fois de plus, le soupçon de sa vérité intérieure lui fait quêter une confirmation de son être fantasmé. Mais la brusquerie impliquée par l'idéal de sincérité prôné par Ergaste ${ }^{29}$ le rend cette fois-ci incapable de conformer ses propos à ce que son interlocutrice a besoin d'entendre. Il accumule les bévues: non content d'avouer que son amour actuel équivaut en force à celui qu'il éprouvait pour une de ses maîtresses passées, il s'extasie sur la beauté d'Araminte qu'il insinue supérieure à celle de la Marquise qui plairait davantage. Blessée dans sa vanité, son interlocutrice s'empresse alors de rendre coup pour coup. Son agressivité ne s'explique toutefois pas uniquement par le besoin de conjurer l'angoisse d'être abandonnée, comme le suggérait Verhoeff ${ }^{30}$. La franchise d'Ergaste remet en cause l'image mentale à laquelle elle s'efforçait de croire. Elle la renvoie à la conscience de son narcissisme infondé : la présenter comme comparable revient en effet à lui révéler que son illusion de supériorité repose sur du vide. Ce n'est évidemment pas là ce que dit Ergaste; ce n'est sans doute pas même ce qu'il pense. La Marquise ne s'arrête pas à la 
littéralité de ses propos mais leur confère un contenu implicite conforme à son intuition de sa vérité intime. La fin de la scène le confirme ${ }^{31}$ :

ERGASTE : Quant à vos esprits, le vôtre me parait bien vif, bien sensible, bien délicat.

LA MARQUISE : Vous biaisez ici ; c'est vain et emporté que vous voulez dire. ils conjugueront leurs efforts pour restaurer son narcissisme mis à mal. Illustrant la « conspiration » humaine sur laquelle se fonde la survie sociale pour Nicole ${ }^{41}$, les deux personnages travestiront leur opinion véritable pour n'offrir à la Marquise que le reflet de son moi chimérique.

Survenant la première, Lisette rassérène l'amour-propre de sa maîtresse. Elle discrédite Ergaste par le recours à deux arguments ad hominem qu'elle agence stratégiquement pour accentuer sa dépréciation : celui-ci étant non seulement "étrange " mais aussi "hypocondre", son "témoignage " $^{42}$ ne saurait être recevable. Elle achève d'en contrebalancer l'impact dévastateur par l'invocation répétée de la caution publique : les invités qui étaient venus lui rendre visite auraient convenu du charme de la Marquise avec autant d'unanimité que de sincérité puisqu'ils ne pouvaient deviner que 
leur conversation serait surprise. Lisette travestit ainsi ses affabulations complaisantes sous le masque de l'objectivité. Transparaissant dans le choix de l'argument développé, son habileté se révèle davantage encore dans son mode d'énonciation. Pour se conformer à la "vanité hypocrite ${ }^{43}$ de sa maîtresse, la suivante nuance ses louanges au moment même où elle les formule. Mieux encore, elle pousse la flatterie au point de suggérer que ses compliments restent euphémisés : aux yeux de "toute la terre », la Marquise serait « une des plus jolies femmes de France - (elle lui) épargne le nom de belle $»^{44}$ pour l'inciter à se reconnaître comme telle. En substituant l'insinuation de la qualité à son affirmation, Lisette s'adresse à la Marquise comme elle le faisait ellemême pour refouler la conscience de sa vérité intime: sa duplicité langagière lui permettait de louvoyer pour satisfaire sa vanité tout en se dissimulant son existence. Elle réussit d'autant mieux à berner sa maîtresse avec sa construction d'une bonne foi illusoire que, comme elle l'avait annoncé à Frontin, « elle la loue brusquement du ton dont on querelle $»^{45}$.

31 À la scène suivante, Dorante reprend, avec succès, les mêmes procédés que Lisette pour voler à la rescousse du narcissisme ébranlé de la Marquise. Inversant complètement ses réflexes comportementaux, il se fait faux sincère en travestissant ses flatteries sous le masque de l'objectivitée ${ }^{46}$ :

Vous êtes fort libre de penser de vous ce qu'il vous plaira, je ne m'y oppose point ; mais je ne suis ni un adulateur ni un visionnaire ; j'ai les yeux bons, j'ai le jugement sain, je sais rendre justice, et je soutiens que vous êtes une des femmes du monde la plus aimable, la plus touchante ; je soutiens qu'il n'y aura point de contradiction làdessus; et tout ce qui me fâche en le disant, c'est que je ne saurais le soutenir sans faire l'éloge d'une personne qui m'outrage et que je n'ai nulle envie de louer.

Invoquant la caution publique comme Lisette, Dorante modère les compliments qu'il adresse à la Marquise. Alors qu'à la scène XI il exaltait maladroitement « le pouvoir de (sa) beauté » avec l'« adoration» et la " passion » ${ }^{47}$ qu'elle lui inspirait, il joue à présent le rôle de l'observateur lucide et impartial qui se contente de la reconnaître comme « une des femmes du monde la plus aimable, la plus touchante ». L'évolution du lexique du personnage est ostensible : le superlatif relatif se substitue à l'hyperbole; l'éloge enthousiaste du cœur sincèrement épris cède la place à la duplicité des prétéritions. Dorante a manifestement appris à apprivoiser son discours, comme le confirme la fin de la scène. Il pousse l'habileté au point de quereller la Marquise pour certains défauts mineurs ; en particulier, sa négligence et la gaucherie de son maintien. De la sorte, loin de la rebuter, il la transporte d'aise. Les Ecrits de morale de Nicole nous fournissent là encore l'explication de ce paradoxe apparent ${ }^{48}$ :

Nous avons deux sortes de défauts : les uns qui sont l'objet de notre attache, et que nous ne voulons pas reconnaître pour défauts, de peur d'être obligés de nous en défaire; les autres que nous condamnons de bonne foi, auxquels nous avons peu d'attache, et dont nous voudrions bien être délivrés. On choisit donc d'abord pour confesseur celui dont on croit qu'il jugera à peu près de nous comme nous désirons qu'il en juge. Ensuite l'on fait comme une espèce de convention et de partage avec lui. On lui abandonne les défauts que l'on n'aime point, on trouve bon qu'il les reprenne comme on les reprend soi-même; mais pour les objets des véritables passions, on ne les soumet guère à la censure d'un confesseur, et on ne le choisit même que dans la pensée qu'il n'y touchera point.

On justifie ainsi premièrement ses passions à soi-même, et l'on cherche ensuite quelque confesseur qui soit disposé à les justifier. En un mot nous voulons en eux une lumière qui n'aille pas plus loin que la nôtre, et qui s'y conforme en tout. C'est- 
à-dire que nous voulons qu'ils approuvent et qu'ils condamnent ce que nous approuvons et ce que nous condamnons nous-mêmes.

La Marquise applaudit Dorante pour sa conformité à sa mauvaise foi : incarnant sa vanité hypocrite, il censure sa vérité intérieure pour ne refléter que son moi fantasmatique. Son jeu de rôle lui permet ainsi de remporter l'enjeu : la main de la Marquise. Comme le résume Jean Dagen dans une contribution essentielle, « le pouvoir attractif de l'âme est [...] inversement proportionnel à sa visibilité, directement proportionnel à sa discrétion ${ }^{49}$; c'est parce qu'il se respecte enfin au lieu de se laisser humilier par son amour et celle qui l'inspire que Dorante parvient aussi à la séduire. Insolitement, sa réussite se révèle donc déterminée par l'expansion de son amourpropre.

4 Le dénouement des Sincères laisse dubitatif. Dorante aime-t-il toujours la Marquise qu'il parvient à épouser? Ne réussit-il à l'épouser que pour avoir cessé de l'aimer? J'ai tendance à croire, pour ma part, que l'évolution de la pratique discursive du personnage fut permise par la modification de ses sentiments. Quoique la Marquise ait soupçonné le contraire au début de la pièce, les «exagérations ${ }^{50}$ de Dorante manifesteraient paradoxalement sa sincérité : un amoureux sincèrement épris transfigure l'objet de son amour et se magnifie lui-même en héros de roman. L'apprentissage du maniement du langage connote inversement la perte de l'innocence et de l'authenticité : Dorante peut réfléchir à ses paroles parce qu'il ne les sent plus.

Les Sincères de Marivaux représenteraient donc conjointement l'altération inévitable de la bonne foi par la duplicité... et le besoin vital de mauvaise foi. La vanité rend la vérité insupportable. La société ne paraît pouvoir survivre que grâce à la complaisance de ses membres, c'est-à-dire à leurs mensonges réciproques ${ }^{51}$ : «aveugles volontaires, (les hommes) haïssent et fuient la lumière, et (...) ne travaillent à rien davantage qu'à se tromper eux-mêmes, et s'entretenir dans l'illusion. "

BIBLIOGRAPHIE

\section{Textes supports}

Marivaux, Les Sincères, dans Théâtre complet, Paris, Seuil, [1964], 2002, p. 480-492.

Molière, Le Misanthrope, dans Euvres complètes, Paris, Seuil, 2002, p. 323-345.

\section{Divers}

Béatrice Agenin et Philippe Miquel, Les Sincères, Société de promotion audiovisuelle théâtrale, 2004. 
Jean Dagen, « Séductions d'âmes chez Marivaux », dans Littérature et séduction, mélanges en l'honneur de Laurent Versini, édités par Roger Marchal et François Moureau, Paris, Klincksieck, 1997, p. 309-320.

Patrick, Dandrey, « Célimène portraitiste. Du salon mondain à l'atelier du Peintre », dans Le salon et la scène : comédie et mondanité au XVII ${ }^{e}$ siècle, Littératures classiques $n^{\circ} 58$, sous la direction de Gabriel Conesa et Véronique Sternberg, Paris, Champion, 2006, p. 11-21.

Charles Rivière, Dufresny, L'Esprit de contradiction, dans Théâtre du XVII ${ }^{e ̀ m e}$ siècle, tome III, édition de Jacques Truchet et André Blanc, Paris, Gallimard, Bibliothèque de la Pléiade, 1992, p. 155-174.

Michel Gilot, « Du Jeu de l'Amour et du hasard aux Fausses confidences, remarques sur l'évolution du théâtre de Marivaux ", dans Vérités à la Marivaux, collectif publié sous la direction de Raymond Joly, Université Laval, Etudes littéraires, n²4, 1991, p. 9-18

Marivaux, Les Acteurs de bonne foi, dans Théâtre complet, Paris, Seuil, 2002, p. 558-567.

Marivaux, Le Cabinet du philosophe, dans Journaux et Cuvres diverses, édition de Frédéric Deloffre et Michel Gilot, Paris, Garnier, Classiques Garnier, p. 389-435.

Pierre Nicole, De la Connaissance de soi-même, dans Essais de morale, édités par Laurent Thirouin, Paris, Presses Universitaires de France, 1999, 440 p.

John O'Neal, « La confusion subversive dans La Double Inconstance », dans Marivaux subversif?, actes du colloque réunis par Franck Salaün, Paris, Desjonquères, 2003, p. 286-292

Anne-Marie Paillet-Guth, « Marivaux, Crébillon et la mauvaise foi : les détours d'une déclaration d'amour ", dans Revue Marivaux, $\mathrm{n}^{\circ}$ 1, coordonné par Françoise Rubellin, Paris, Société Marivaux, mai 1990, p. 53-64.

Martin Riegel, Jean-Christophe Pellat et René Rioul, Grammaire méthodique du français, Paris, PUF, Quadrige, [1991], 2001.

John Kristian Sanaker, Le Discours mal apprivoisé : essai sur le dialogue chez Marivaux, Oslo, Société Nouvelle Didier Erudition, 1987.

Jean-Paul Sartre, L'Être et le Néant : essai d'ontologie phénoménologique, Paris, Gallimard, Tel, 2007.

Pierre, Schoentjes, Poétique de l'ironie, Paris, Seuil, 2001.

Han Verhoeff, Marivaux ou le dialogue avec la femme, Orléans, Paradigme, 1994.

\section{NOTES}

1. John Kristian Sanaker, Le Discours mal apprivoisé : essai sur le dialogue chez Marivaux, Oslo, Société Nouvelle Didier Erudition, 1987, 133 p.

2. Anne-Marie Paillet-Guth, "Marivaux, Crébillon et la mauvaise foi: les détours d'une déclaration d'amour ", dans Revue Marivaux, $n^{\circ}$ 1, coordonné par Françoise Rubellin, Paris, Société Marivaux, mai 1990, p. 53-64.

3. Michel Gilot, « Du Jeu de l'Amour et du hasard aux Fausses confidences, Remarques sur l'évolution du théâtre de Marivaux ", dans Vérités à la Marivaux, collectif publié sous la direction de Raymond Joly, Université Laval, Etudes littéraires, n² 24, 1991, p. 9-18.

4. John O’Neal, «La Confusion subversive dans La Double Inconstance », dans Marivaux subversif?, actes du colloque réunis par Franck Salaün, Paris, Desjonquères, 2003, p. 286-292.

5. Marivaux, Les Sincères, scène 1, réplique de Lisette, p. 481.

6. Voir la scène V de l'acte III page $337 \mathrm{du}$ Misanthrope. 
7. Molière, Le Misanthrope, acte IV, scène I, p. 338.

8. Molière, Ibidem, acte III, scène IV, page 336.

9. Marivaux, "Le Voyageur dans le Nouveau Monde", dans Le Cabinet du Philosophe, huitième feuille, p. 401.

10. Molière, Le Misanthrope, acte I, scène 1, p. 324.

11. Voir notamment les propos qu'il tient à Philinte à ce sujet ; acte I, scène 1, page 324 .

12. Aristote, Ethique à Nicomaque, introduction, traduction et commentaire par R. Gauthier et J.-Y. Jolil, Louvain-Paris, Nauwelaerts, 1970. Cité par Pierre, Schoentjes, Poétique de l'ironie, Paris, Seuil, 2001, p. 35.

13. Marivaux, L'Indigent philosophe, dans Journaux et Cuvres diverses, sixième feuille, p. 315.

14. Molière, Le Misanthrope, acte II, scène IV, p. 332.

15. Alceste évoque, plus précisément, le «coupable encens de (la) flatterie » des Marquis Clitandre et Acaste. Molière, Ibidem, acte II, scène IV, p. 322.

16. Patrick Dandrey, «Célimène portraitiste. Du salon mondain à l'atelier du Peintre ", dans Le salon et la scène: comédie et mondanité au XVII ${ }^{e}$ siècle, Littératures classiques $n^{\circ} 58$, sous la direction de Gabriel Conesa et Véronique Sternberg, Paris, Champion, 2006, p. 11-21 ; p. 16.

17. Marivaux, Les Sincères, scène IV, p. 483.

18. Marivaux, Op. cit., scène IV, p. 483.

19. Marivaux, Les Acteurs de bonne foi, scène XII, tirade de Blaise, p. 566 : «Et par-dessus, on se raille de ma personne dans ce peste de jeu-là, noute maîtresse ; Colette y fait semblant d'avoir le cœur tendre pour Monsieur Merlin, Monsieur Merlin de li céder le sien; et, maugré la comédie, tout ça est vrai, noute maîtresse ; car ils font semblant de faire semblant. "

20. La source de la célèbre expression utilisée par Marivaux dans Les Acteurs serait une réplique de Lucas, paysan de L'Esprit de contradiction, comédie en un acte de Dufresny, représentée pour la première fois le 27 août 1700. Voir, dans l'édition de Truchet, la scène XII page 167 : «LUCAS : je me sis douté qu'ou maniganciais l'amour ensemble, \& que vous faisiais semblant de faire semblant. "

21. Marivaux, Les Sincères, scène IV, p. 484.

22. Pierre Nicole, De la connaissance de soi-même, dans Essais de morale, édité par Laurent Thirouin, Paris, PUF, 1999, p. 323.

23. Jean-Paul Sartre, L'Etre et le néant: essai d'ontologie phénoménologique, Paris, Gallimard, Tel, 2007, p. 83.

24. Jean-Paul Sartre, Ibidem, p. 87.

25. Sartre, Op. cit., p. 87 : « Le seul plan sur lequel nous pouvons situer le refus du sujet, c'est celui de la censure. Elle seule peut saisir les questions ou les révélations du psychanalyste comme s'approchant de plus ou moins près des tendances réelles qu'elle s'applique à refouler, elle seule parce qu'elle est seule à savoir ce qu'elle refoule. »

26. Nicole, Op. cit.; p. 312.

27. Marivaux, Les Sincères, scène IV, p. 483.

28. Marivaux, Op. cit., scène VI, p. 484.

29. Il le reconnaît lui-même : « Le plus grand défaut de ma sincérité, c'est qu'elle est trop forte. » Ibidem, p. 487.

30. Han Verhoeff, Marivaux ou le dialogue avec la femme, Orléans, Paradigme, 1994, 272 p. Voir, en particulier, la sixième section de son second chapitre : « Vanité et angoisse d'abandon », p. 7-9.

31. Marivaux, Les Sincères, scène XII, p. 488.

32. Voir Martin Riegel, Jean-Christophe Pellat et René Rioul, Grammaire méthodique du français, Paris, PUF, Quadrige, [1991], 2001, p. 585. L'« acte perlocutionnaire (ou perlocutoire) » se définit comme «l'effet produit par l'acte illocutionnaire sur l'allocutaire. Il n'est pas prévu par la convention, mais permet d'évaluer la réussite ou l'échec de l'acte illocutionnaire suivant les réactions de l'allocutaire. » 
33. Voir l'aparté de la Marquise à la scène XV, p. 489 : « .... Assurément, s'il dit vrai, je ne suis pas une jolie personne.»

34. Marivaux, Les Sincères, scène XV, p. 489.

35. Délaissée par son soupirant au profit d'une femme plus jeune, la veuve consulte un magicien pour connaître sa rivale et comprendre les raisons de son inconstance. Mais à peine l'a-t-elle vu dans le miroir magique qu'elle l'accable de son mépris. Le sorcier décide alors de la soumettre à une épreuve : feignant de s'être trompé, il lui donne à voir son propre reflet rajeuni dans le miroir. La veuve ne se reconnaît pas et se déchaîne contre son image avec une acrimonie au moins égale. Lui dévoilant la vérité, le magicien la confronte alors à son mensonge sur ellemême. Mais -mauvaise foi oblige !- la veuve refoule la conscience de sa duplicité.

Marivaux, Le Cabinet du philosophe, dixième feuille, dans Journaux et CEuvres diverses, pp. 418-426.

36. Marivaux, Ibidem, p. 426.

37. Les Sincères; L'Epreuve; réalisés par Philippe Miquel et Béatrice Agenin, Paris, Société de promotion audiovisuelle théâtrale, 2004.

38. Marivaux, Les Sincères, scène XII, p. 487.

39. Marivaux, Ibidem, scène XV, p. 489.

40. Nicole, De la Connaissance de soi-même, dans Op. cit., p. 324.

41. Nicole, Ibidem, p. 321.

42. Marivaux, Op. cit., scène XV, p. 489.

43. Marivaux, Op. cit., scène I, p. 481.

44. Marivaux, Op. cit., scène XV p. 489.

45. Marivaux, Les Sincères, scène I, p. 481.

46. Marivaux, Ibidem, scène XVI, p. 489-490.

47. Marivaux, Op. cit., scène XI, p. 484.

48. Pierre Nicole, De la connaissance de soi-même, chapitre $\mathrm{X}$ : «Qu'on se sert souvent des confesseurs pour s'autoriser dans ses passions », p. 364-365.

49. Jean Dagen, «Séductions d'âmes chez Marivaux ", dans Littérature et séduction, mélanges en l'honneur de Laurent Versini, édités par Roger Marchal et François Moureau, Paris, Klincksieck, 1997, p. 309-320; p. 314.

50. Marivaux, Les Sincères, scène XI, p. 486.

51. Nicole, Op. cit., «Chapitre VI : En quoi consiste l'amour naturel que l'on dit que l'homme a pour la vérité, et quel usage on en fait », p. 325.

INDEX

oeuvrecitee Sincères (Les)

\section{AUTEURS}

BEYA DHRAÏEFF

Ancienne élève de l'Ecole Normale Supérieure, Doctorante à l'Université de la Sorbonne Nouvelle - Paris III 